\title{
Novel Modifications for a Virtual Interview Visit to Simulate the Traditional, Live, Site Visit for GI Fellowship Applicants for an Academic GI Fellowship Program Due to the COVID-19 Pandemic
}

\author{
Mitchell S. Cappell ${ }^{1,2}$
}

Received: 4 November 2020 / Accepted: 20 November 2020 / Published online: 26 January 2021

(c) Springer Science+Business Media, LLC, part of Springer Nature 2021

Keywords Gastroenterology fellowship match · Interview process · COVID-19 · Virtual interviews · GI fellowship · Academic programs

\section{Study Highlights}

Previously known:

- Due to COVID-19 pandemic, virtual interviews have replaced live, on-site, interviews for GI fellowship applicants.

What is new here:

- Novel features recommended for the virtual interview experience to closely resemble the live, on-site visit: virtual guided hospital tours, virtual program discussion/ presentation, confidential virtual meeting of interviewees with current GI fellows, and early involvement of information technologists to set up this virtual program to avoid costly errors.

\section{To the Editor}

To reduce exposure to COVID-19 infection during the current epidemic, we are now replacing the live, onsite, interview process for first-year GI (gastroenterology)

Mitchell S. Cappell

Mitchell.Cappell@Beaumont.edu; mscappell@yahoo.com

1 Division of Gastroenterology and Hepatology, Department of Medicine, William Beaumont Hospital at Royal Oak, MOB \#602, 3535 W. Thirteen Mile Rd, Royal Oak, MI 48073, USA

2 Division of Gastroenterology and Hepatology, Department of Medicine, Oakland University William Beaumont School of Medicine, 3535 W. Thirteen Mile Rd, Royal Oak, MI 48073, USA fellowship applicants, August-November 2020, at William Beaumont Hospital, Royal Oak, with virtual visits and interviews. The traditional site visit includes, aside from applicant interviews, guided hospital tours, a live presentation of the fellowship program, and confidential conversations between interviewees and current GI fellows. This work proposes the novel recommendation to replace all these traditional live interview visit events with virtual events to enhance the virtual visit experience.

This institution is a large, tertiary, academic hospital with a GI fellowship continuously accredited since 1973 by the American College of Graduate Medicine Education, without current program citations, housed in a large academic GI division (36 GI attendings), with a very busy GI endoscopy suite (25,000 endoscopies/annum). The division had 382 applications as of August 20, 2020, the cutoff date for application consideration, for two available first-year fellowship positions. The author claims expertise in GI fellowship program administration and applicant selection, including several publications on this subject; experience as GI fellowship program director at two academic institutions, 2003-current; experience as associate GI fellowship program director at three institutions, 1995-2003; experience as Chief of Gastroenterology at an academic hospital, 2006-2019; and as consultant to United States Food and Drug Administration (FDA) Advisory Committee on Gastrointestinal Drugs, 2014-2019.

Benefits of virtual interviews include: applicants can interview remotely without travel time and without travel expenses incurred by visiting our hospital and staying overnight at a neighborhood hotel. Also, scheduling candidates for virtual interviews, rather than live interviews, is simpler 
because live interviews generally require coordinating consecutive interviews to avoid delays between interviews, whereas virtual interviews can be set up individually at any mutually agreeable time.

Disadvantages of virtual interviews, with proposed remedies, include: First, the ability of GI fellowship applicants to interview without travel costs and travel time may render applicants more willing to interview at fellowships of only marginal interest ("safe programs"). Indeed, $100 \%$ of selected interview candidates accepted our interview offers this year, versus only about $80 \%$ in previous years. This may result in ranking interviewed applicants who have strong credentials but little interest in our fellowship program. To compensate for this effect, we increased the interviewee pool to 26 from 22, previously. Second, previously during live, on-site, interviews, fellowship applicants enjoyed a live, guided tour of the hospital during the visit conducted by a current gastroenterology fellow, which included the GI endoscopy suite, conference room, clinic, hospital library, intensive care room, on-call facilities, fellows' offices, and hospital cafeteria. We created an 8 min videotaped guided tour, distributed privately by Internet, to substitute for this live experience. To minimize costs, a GI fellow and the GI fellowship program director acted as videographer and tour guide, respectively. Third, previous live confidential discussions between applicants and GI fellows during the hospital tour and a complementary lunch were recreated by virtual discussions between them over the internet. Access to this meeting must be denied to GI faculty to maintain confidentiality. Fourth, early involvement of information technologists is critical to set up virtual interviews and to teach attending faculty about this technology to avoid costly mistakes that we encountered early in this process. These recommendations may improve the interview experience for interviewees and interviewers to simulate the live experience and may improve GI patient care by matching the skills and expectations of GI fellowship applicants with the local hospital needs, which depend on hospital size, neighborhood versus academic hospital, endoscopy volume, endoscopy referral patterns (e.g., tertiary versus primary care hospital), local demographics, and public versus private hospitals.

Author's contribution Dr. Cappell conceived and wrote the entire letter to the editor.

Funding None.

\section{Compliance with Ethical Standards}

Conflict of interest None for the author. Dr. Cappell, as a consultant of the United States Food and Drug Administration (FDA) Advisory Committee for Gastrointestinal Drugs, 2014-2019, affirms that this paper does not discuss any proprietary confidential pharmaceutical data submitted to the FDA. Dr. Cappell was a member of the speaker's bureau for AstraZeneca and Daiichi Sankyo, co-marketers of Movantik until $>2$ years ago. Dr. Cappell received one-time consulting fees from Mallinckrodt and Shire $>2$ years ago. This work does not discuss any drug manufactured or marketed by AstraZeneca, Daiichi Sankyo, Mallinckrodt, or Shire.

Publisher's Note Springer Nature remains neutral with regard to jurisdictional claims in published maps and institutional affiliations. 